\title{
Eliminating the Effect of Common-Mode Voltage on an Open-End Winding PMSM Based on Model Predictive Torque Control
}

\author{
Zhiming Lin $\mathbb{D},{ }^{1}$ Bicheng Lei $\mathbb{D},{ }^{1}$ Lingwei $W u\left(\mathbb{D},{ }^{1,2}\right.$ and Pan Mei $\mathbb{D}^{1}$ \\ ${ }^{1}$ College of Electronic and Information Engineering, Taizhou University, Taizhou, Zhejiang, China \\ ${ }^{2}$ College of Automation Engineering, Nanjing University of Aeronautics and Astronautics, Nanjing, China
}

Correspondence should be addressed to Bicheng Lei; 52328173@qq.com

Received 25 January 2021; Revised 24 March 2021; Accepted 18 April 2021; Published 7 June 2021

Academic Editor: Xiaodong Sun

Copyright $(0) 2021$ Zhiming Lin et al. This is an open access article distributed under the Creative Commons Attribution License, which permits unrestricted use, distribution, and reproduction in any medium, provided the original work is properly cited.

In the open-end winding permanent magnet synchronous motor (OEW-PMSM) with common DC link, the common-mode voltage (CMV) will cause leakage current and zero-sequence current, which will lead to the decrease in the system life and efficiency. To solve this problem, the loop characteristics of leakage current and zero-sequence current were analyzed, and the condition for eliminating the leakage current and the zero-sequence current was deduced. Then, the CMV of the voltage vectors for the OEW-PMSM system was calculated, and the appropriate voltage vectors satisfying the conditions were selected to form the control vector sets. Combined with the model predictive torque control (MPTC), a cost function without the weight factor was proposed. The voltage vector sets were predicted by the cost function. The optimal voltage vectors were selected to control the OEW-PMSM, which can eliminate the leakage current and the zero-sequence current caused by the CMV. The effectiveness of the proposed method was verified by the simulation results.

\section{Introduction}

Due to the advantages of high power, more voltage vectors, and high fault tolerance, open-end winding (OEW) motors have attracted wide attention in high-power, large-capacity, and vehicle applications [1-4]. In the OEW system, the inverter connected to both ends of the open winding often uses a single common DC power supply instead of two isolated power supplies for lower costs [2-4].

Since the inverter uses the pulse-width modulation (PWM) technology to drive the motor in the OEW, there exists common-mode voltage (CMV) in the motor winding. Through parasitic capacitive impedance, high-frequency CMV generates leakage current which will damage the shaft and bearing $[5,6]$. Furthermore, CMV will cause zerosequence current and reduce the efficiency of the OEW system because of being a zero-sequence loop in the common DC power supply of the OEW system $[1,2,7,8]$.
Therefore, zero-sequence current (ZSC) and leakage current caused by CMV should be eliminated in the system.

As CMV frequency reduces, the leakage current of the system will also be reduced. A method proposed in $[9,10]$ uses three vectors with the same CMV to synthesize the reference voltage to reduce the CMV frequency. An active zero-state PWM method can reduce the CMV frequency by replacing the zero vector with two vectors in the opposite direction [11]. Based on the principle of volt-second, Kalaiselvi and Srinivas [12] proposed a hybrid PWM switching method to reduce CMV and leakage current for the OEW system. All these methods have a vector modulation process, which is complex.

In addition to the vector synthesis technique, there are also other methods to solve this problem. A multilevel method has been presented to eliminate CMV and leakage current in [13-15]. Chokes are used as filters to eliminate leakage current caused by CMV in [16-18]. Some protective 
aluminium films are added between the stator and the rotor to prevent leakage current caused by CMV in [19]. All these methods need extra hardware.

As for ZSC reducing in OEW, a modulation for the considered topology which is able to eliminate the ZSC is proposed in [7]. The duration time of the active voltage vectors is redistributed to the suppression of ZSC in [8]. A space-vector PWM scheme with the capability of zerosequence voltage control is proposed to eliminate the ZSC in [4].

These aforementioned methods can only eliminate ZSC or leakage current, but cannot eliminate both at the same time. Due to different mechanisms of leakage current and ZSC generated by CMV, few literature studies can simultaneously eliminate the influence of CMV on leakage current and ZSC.

A method to simultaneously eliminate the influence of CMV on ZSC and leakage current in the OEW-PMSM is proposed in this paper. Different from the aforementioned method that requires vector modulation or additional hardware, this method only needs to select a suitable vector set according to certain conditions and then use the selected vector set in MPTC to control the OEW system. Meanwhile, when applying MPTC, a cost function without weight factors is proposed. The method is simple and easy to implement, and the effectiveness and feasibility of the proposed method are verified by simulation.

\section{Model of the OEW-PMSM Drive System}

The structure of the OEW-PMSM system with common DC link is shown in Figure 1. The system is fed by two-level inverters VSI1 and VSI2. Because of the existing zerosequence loop, different voltages of CMV between the two inverters will produce zero-sequence current $i_{0}$. At the same time, the CMV with high frequency can produce leakage current $i_{L}$ because of the existing parasitic capacitive impedance between the phase windings, motor frame, and bearing [5].

The stator voltage equations of the OEW-PMSM can be expressed as

$$
\begin{aligned}
& u_{d}=R_{s} i_{d}+L_{d} \frac{\mathrm{d} i_{d}}{\mathrm{~d} t}-\omega_{r} L_{q} i_{q}, \\
& u_{q}=R_{s} i_{q}+L_{q} \frac{\mathrm{d} i_{q}}{\mathrm{~d} t}+\omega_{r} L_{d} i_{d}+\omega_{r} \psi_{f}, \\
& u_{0}=R_{s} i_{0}+L_{0} \frac{\mathrm{d} i_{0}}{\mathrm{~d} t},
\end{aligned}
$$

where $u_{d}, u_{q}, u_{0}, i_{d}, i_{q}, i_{0}, L_{d}, L_{q}$, and $L_{0}$ are the stator voltage, current, and inductance components in the $d_{-}, q^{-}$, and 0 axis, respectively, $R_{s}$ is the stator resistance, $\omega_{r}$ is the electrical angular speed, and $\Psi_{f}$ is the rotor flux linkage.

The flux and torque equations of the OEW-PMSM are expressed as

$$
\begin{aligned}
& \psi_{d}=L_{d} i_{d}+\psi_{f}, \\
& \psi_{q}=L_{q} i_{q}, \\
& T_{e}=\frac{3}{2} n_{p}\left(\psi_{d} i_{q}-\psi_{q} i_{d}\right) .
\end{aligned}
$$

It is easy to obtain the phase voltages $u_{a}, u_{b}$, and $u_{c}$ across the phase winding from Figure 1 as

$$
\begin{aligned}
& u_{a}=u_{a 1}-u_{a 2}=\left(S_{a 1}-S_{a 2}\right) U_{\mathrm{dc}} \\
& u_{b}=u_{b 1}-u_{b 2}=\left(S_{b 1}-S_{b 2}\right) U_{\mathrm{dc}} \\
& u_{c}=u_{c 1}-u_{c 2}=\left(S_{c 1}-S_{c 2}\right) U_{\mathrm{dc}}
\end{aligned}
$$

where $\left(S_{a 1}, S_{a 2}, S_{b 1}, S_{a 2}, S_{c 1}, S_{c 2}\right) \in(0,1)$ are the switching state of six legs. $S_{a 1}=0$ indicates that the upper leg switch is off and the lower leg switch is on, and $u_{a 1}=0$. If $S_{a 1}=1$, the leg switch state is opposite, and $u_{a 1}=U_{\mathrm{dc}}$. The other legs are similar.

The CMV of the two inverters is defined as [7]

$$
\begin{aligned}
& \mathrm{CMV} 1=\frac{u_{a 1}+u_{b 1}+u_{c 1}}{3}, \\
& \mathrm{CMV} 2=\frac{u_{a 2}+u_{b 2}+u_{c 2}}{3} .
\end{aligned}
$$

Then, the voltage of the zero-sequence loop can be further expressed as [12]

$$
u_{0}=\frac{1}{3}\left(u_{a}+u_{b}+u_{c}\right)=\mathrm{CMV} 1-\mathrm{CMV} 2
$$

The voltage of the leakage current loop is defined as [12]

$$
u_{L}=\frac{\mathrm{CMV} 1+\mathrm{CMV} 2}{2}
$$

For parasitic capacitive impedance in the leakage current loop, as it is known, current $i_{L}$ can be expressed as

$$
i_{L}=C_{L} \frac{\mathrm{d} u_{L}}{\mathrm{~d} t}
$$

Equations (2) and (6)-(8) show that, in order to eliminate the influence of $\mathrm{CMV}$ on the system, for the zerosequence loop, when CMV1 $=\mathrm{CMV} 2, u_{0}=0$, and CMV will not generate zero-sequence current $i_{0}$. And for parasitic capacitive impedance $C_{L}$ of the leakage current loop, as long as $u_{L}=C$ ( $C$ is constant $)$, it means that the frequency of $u_{L}$ is zero, and CMV will not generate current $i_{L}$ on $C_{L}$.

Therefore, the condition of eliminating the influence of CMV on both zero-sequence loop and leakage current loop is obtained as

$$
\begin{gathered}
u_{L}=C, \\
u_{0}=0,
\end{gathered}
$$

where $C$ is a constant. 


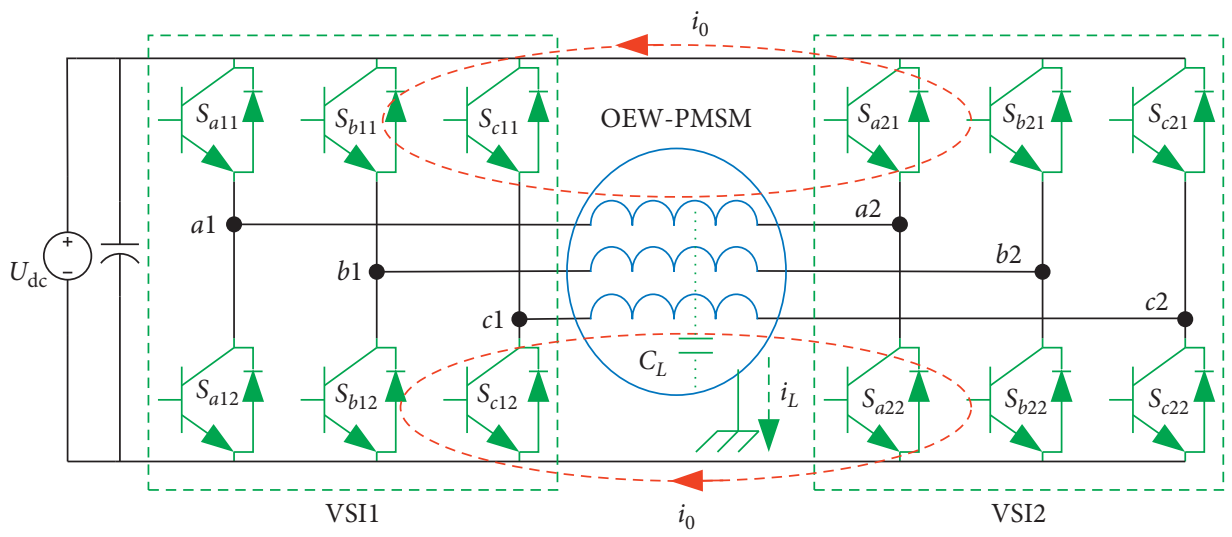

Figure 1: Block diagram of the OEW-PMSM with common DC link.

\section{Voltage Vector Selection}

In Figure 1, each inverter has eight voltage vectors, including six nonzero voltage vectors and two zero voltage vectors. The voltage vectors of the two inverters can be expressed by (10) and (11), and the amplitude of six nonzero voltage vectors is $2 U_{\mathrm{dc}} / 3$.

$$
\begin{aligned}
& U_{s 1}=\frac{2 U_{\mathrm{dc}}}{3}\left(S_{a 1}+S_{b 1} e^{j(2 / 3) \pi}+S_{c 1} e^{j(4 / 3) \pi}\right), \\
& U_{s 2}=\frac{2 U_{d c}}{3}\left(S_{a 2}+S_{b 2} e^{j(2 / 3) \pi}+S_{c 2} e^{j(4 / 3) \pi}\right) .
\end{aligned}
$$

The distribution of space voltage vectors is shown in Figure 2 for each inverter.

From the structure of the two inverters in Figure 1, it can be seen that the synthesized voltage vectors of the two inverters can be expressed as

$$
U_{s}=U_{s 1}-U_{s 2} \text {. }
$$

The synthesized voltage vectors of the two inverters are 64 vectors, including 56 nonzero voltage vectors and 8 zero voltage vectors. The synthesized voltage vectors can be seen in Figure 3, where the amplitude of six large voltage vectors is $4 U_{\mathrm{dc}} / 3$, the amplitude of twelve medium voltage vectors is $2 \sqrt{3} U_{\mathrm{dc}} / 3$, and the amplitude of thirty-six small voltage vectors is $2 U_{\mathrm{dc}} / 3$.

For each voltage vector in Figure 3, CMV1 and CMV2 can be calculated according to (5), and then $u_{\mathrm{L}}$ and $u_{0}$ can be calculated according to (6) and (7). For example, CMV1 and CMV2 of voltage vector $4-1^{\prime}$ are calculated as follows:

$$
\begin{aligned}
& \text { CMV1 }=\frac{S_{a 1}+S_{b 1}+S_{c 1}}{3} U_{\mathrm{dc}}=\frac{1+0+0}{3} U_{\mathrm{dc}}=\frac{U_{\mathrm{dc}}}{3}, \\
& \mathrm{CMV} 2=\frac{S_{a 2}+S_{b 2}+S_{c 2}}{3} U_{\mathrm{dc}}=\frac{0+0+1}{3} U_{\mathrm{dc}}=\frac{U_{\mathrm{dc}}}{3} .
\end{aligned}
$$

Then, $u_{L}$ and $u_{0}$ are calculated as follows:

$$
\begin{aligned}
& u_{0}=\mathrm{CMV} 1-\mathrm{CMV} 2=\frac{U_{\mathrm{dc}}}{3}-\frac{U_{\mathrm{dc}}}{3}=0, \\
& u_{L}=\frac{\mathrm{CMV} 1+\mathrm{CMV} 2}{2}=\frac{1}{2}\left(\frac{U_{\mathrm{dc}}}{3}+\frac{U_{\mathrm{dc}}}{3}\right)=\frac{U_{\mathrm{dc}}}{3} .
\end{aligned}
$$

In order to obtain the voltage vector sets that meet condition (9), $u_{L}$ and $u_{0}$ of each voltage vector in Figure 3 are calculated firstly. Secondly, the voltage vectors with $u_{0}=0$ are chosen, and then they are classified according to the value of $u_{L}$. Finally, two voltage vector sets meeting condition (9) can be obtained as shown in Table 1. These two voltage vector sets include nonzero medium voltage vectors (row 1,3) and zero voltage vectors (row 2, 4). These six nonzero medium voltage vectors in each set can form a voltage vector circle. Therefore, these two voltage vector sets can be used to control the OEW-PMSM.

From Table 1, it can been seen that set-I consists of nonzero medium voltage vectors in row 1 and zero voltage vectors in row 2 , in which $u_{0}$ is always zero and $u_{L}$ is always $2 U_{\mathrm{dc}} / 3$. Set-II consists of nonzero medium voltage vectors in row 3 and zero voltage vectors in row 4 , in which $u_{0}$ is always zero and $u_{L}$ is always $U_{\mathrm{dc}} / 3$.

\section{Model Predictive Torque Control}

Because of the fast dynamic response and flexible cost function configuration, the model predictive control (MPC) has developed rapidly in recent years $[20,21]$. Meanwhile, MPC has also been used to eliminate ZSC in [22, 23].

In order to achieve better performance, voltage vector sets I and II are applied to MPTC to control the OEWPMSM system. According to the forward Euler method, the prediction model of the OEW-PMSM can be obtained. The current prediction equations can be expressed as 


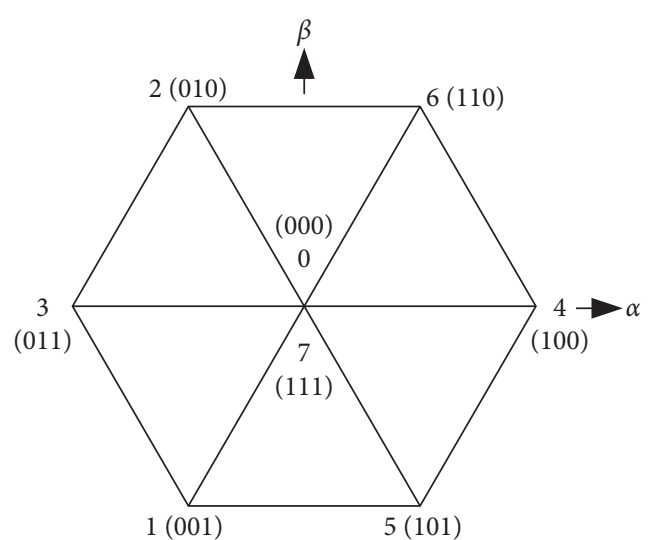

(a)

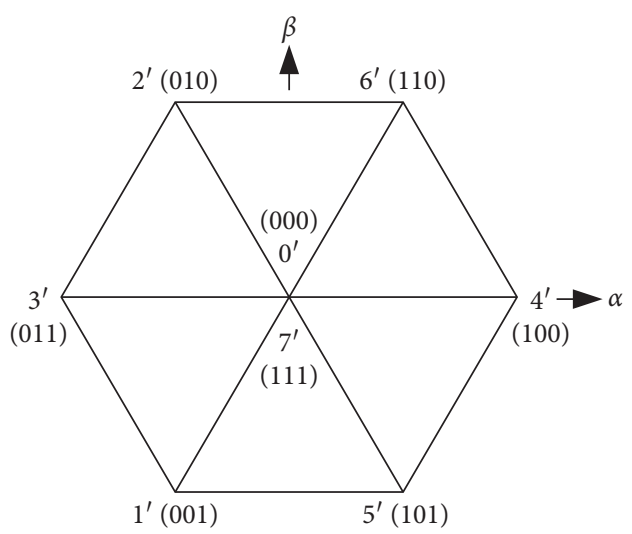

(b)

FIGURE 2: Space voltage vector of two-level inverters. (a) VSI1. (b) VSI2.

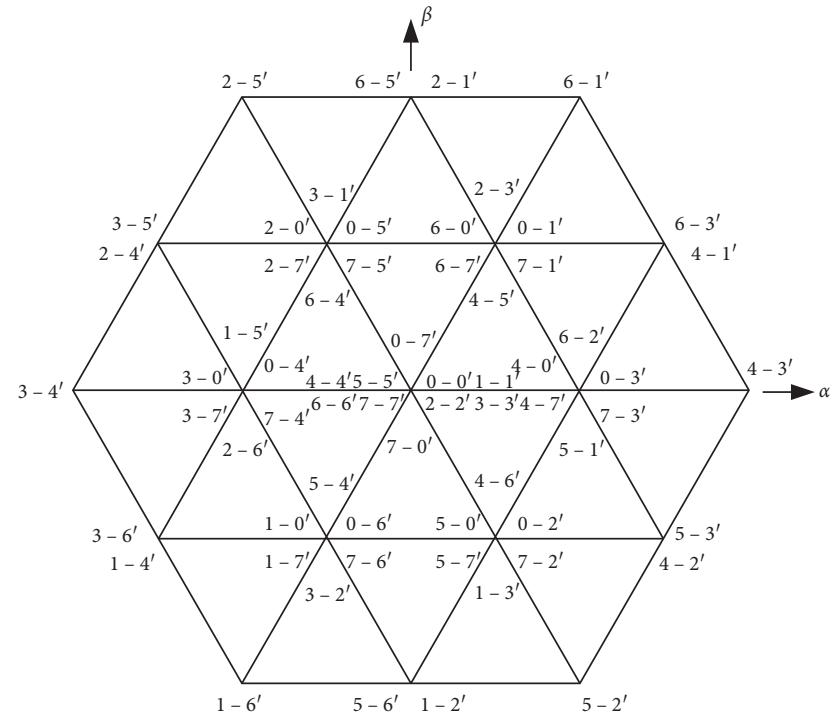

Figure 3: Double inverters synthesize space voltage vectors.

$$
\begin{aligned}
& i_{d}^{k+1}=\left(1-\frac{R_{S}}{L_{d}} T_{s}\right) i_{d}^{k}+\frac{u_{d}^{k}}{L_{d}} T_{s}+\omega_{r} L \frac{i_{q}^{k}}{L_{d}} T_{s}, \\
& i_{q}^{k+1}=\left(1-\frac{R_{S}}{L_{q}} T_{s}\right) i_{q}^{k}+\frac{u_{q}^{k}}{L_{q}} T_{s}-\omega_{r} L_{d} \frac{i_{d}^{k}}{L_{q}} T_{s}-\omega_{r} \frac{\psi_{f}}{L_{q}} T_{s} .
\end{aligned}
$$
follows:

Meanwhile, flux and torque prediction equations are as

$$
\begin{aligned}
& \psi_{d}^{k+1}=L_{d} i_{d}^{k+1}+\psi_{f}, \\
& \psi_{q}^{k+1}=L_{q} i_{q}^{k+1}, \\
& T_{e}^{k+1}=\frac{3}{2} n_{p}\left(\psi_{d}^{k+1} i_{q}^{k+1}-\psi_{q}^{k+1} i_{d}^{k+1}\right) .
\end{aligned}
$$

\begin{tabular}{|c|c|c|c|c|c|c|}
\hline Row & Vector & CMV1 & CMV2 & $u_{0}$ & $u_{L}$ & Set \\
\hline 1 & $\begin{array}{l}6-3^{\prime}, 6-5^{\prime}, 3-5^{\prime} \\
3-6^{\prime}, 5-6^{\prime}, 5-3^{\prime}\end{array}$ & $2 U_{\mathrm{dc}} / 3$ & $2 U_{\mathrm{dc}} / 3$ & 0 & $2 U_{\mathrm{dc}} / 3$ & I \\
\hline 2 & $6-6^{\prime}, 5-5^{\prime}, 3-3^{\prime}$ & $2 U_{\mathrm{dc}} / 3$ & $2 U_{\mathrm{dc}} / 3$ & 0 & $2 U_{\mathrm{dc}} / 3$ & \\
\hline 3 & $\begin{array}{l}4-1^{\prime}, 2-1^{\prime}, 2-4^{\prime} \\
1-4^{\prime}, 1-2^{\prime}, 4-2^{\prime}\end{array}$ & $U_{\mathrm{dc}} / 3$ & $U_{\mathrm{dc}} / 3$ & 0 & $U_{\mathrm{dc}} / 3$ & II \\
\hline 4 & $4-4^{\prime}, 2-2^{\prime}, 1-1^{\prime}$ & $U_{\mathrm{dc}} / 3$ & $U_{\mathrm{dc}} / 3$ & 0 & $U_{\mathrm{dc}} / 3$ & \\
\hline
\end{tabular}

The traditional cost function of MPTC is as follows [22]:
TABLE 1: Voltage vector set (I and II) and CMV analysis.

When (17) is used as the cost function, it is difficult to tune weighting factor $\lambda$ in practical applications. Generally, the value of $\lambda$ is related to $T_{e}^{\text {ref }}$ and $\psi_{s}^{\text {ref }}$. If $T_{e}^{\text {ref }}$ and $\psi_{s}^{\text {ref }}$ change dynamically in the process of application, $\lambda$ should also be changed correspondingly in order to achieve the best performance. In order to solve this problem, a new and simplified cost function is proposed as

$$
C=\left|\frac{T_{e}^{\mathrm{ref}}-T_{e}^{k+1}}{T_{e}^{\mathrm{ref}}}\right|+\left|\frac{\psi_{s}^{\mathrm{ref}}-\psi_{s}^{k+1}}{\psi_{s}^{\mathrm{ref}}}\right| .
$$

There is no weighting factor in (18). Especially, in practical applications, when $T_{e}^{\text {ref }}$ and $\psi_{s}^{\text {ref }}$ change, (18) can adapt automatically without changing. If $T_{e}^{\text {ref }}$ is equal to 0 , it can be replaced by a small number, such as $1 e-3$.

The block diagram of the MPTC for the OEW-PMSM is shown in Figure 4.

The proposed MPTC selects an optimal vector from set-I or set-II according to the minimum value of the cost function (18) in each control cycle. If the optimal vector is a zero voltage vector, it should be selected according to the principle of minimum switching times from set-I or set-II.

\section{Simulation Studies}

The proposed method is verified by simulation with a sampling frequency of $100 \mathrm{kHz}$. In the simulation, six large 


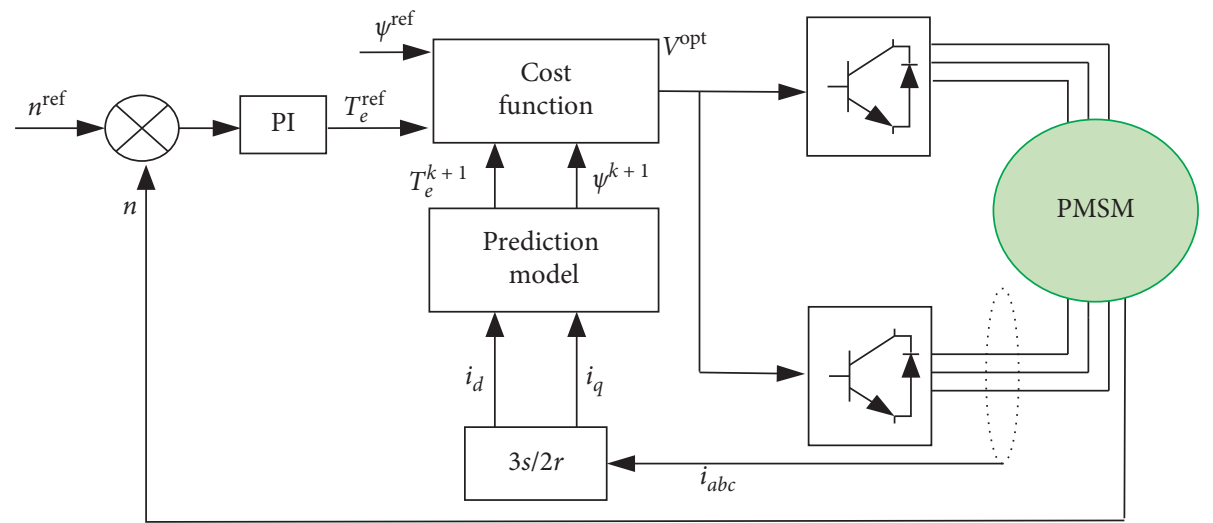

FIgURE 4: Control diagram of the MPTC.

TABLE 2: Voltage vector set-III and CMV analysis.

\begin{tabular}{|c|c|c|c|c|c|}
\hline No. & Vector & CMV1 & CMV2 & $u_{0}$ & $u_{L}$ \\
\hline 1 & $4-3^{\prime}, 2-5^{\prime}, 1-6^{\prime}$ & $U_{\mathrm{dc}} / 3$ & $2 U_{\mathrm{dc}} / 3$ & $-U_{\mathrm{dc}} / 3$ & $U_{\mathrm{dc}} / 2$ \\
\hline 2 & $3-4^{\prime}, 6-1^{\prime}, 5-2^{\prime}$ & $2 U_{\mathrm{dc}} / 3$ & $U_{\mathrm{dc}} / 3$ & $U_{\mathrm{dc}} / 3$ & $U_{\mathrm{dc}} / 2$ \\
\hline 3 & $0-0^{\prime}$ & 0 & 0 & 0 & 0 \\
\hline
\end{tabular}

TABle 3: Parameters of the PMSM.

\begin{tabular}{lc}
\hline Machine parameter & Value \\
\hline$d$-axis inductance $(\mathrm{H})$ & $8.5 e-3$ \\
$q$-axis inductance $(\mathrm{H})$ & $8.5 e-3$ \\
Number of pole pairs & 4 \\
Stator resistance $(\Omega)$ & 1.2 \\
Rated torque $(\mathrm{N} \mathrm{m})$ & 15 \\
Rated speed $(\mathrm{r} / \mathrm{min})$ & 1000 \\
Flux $(\mathrm{Wb})$ & 0.175 \\
$C_{L}$ & $70 \mathrm{pF}$ \\
\hline
\end{tabular}
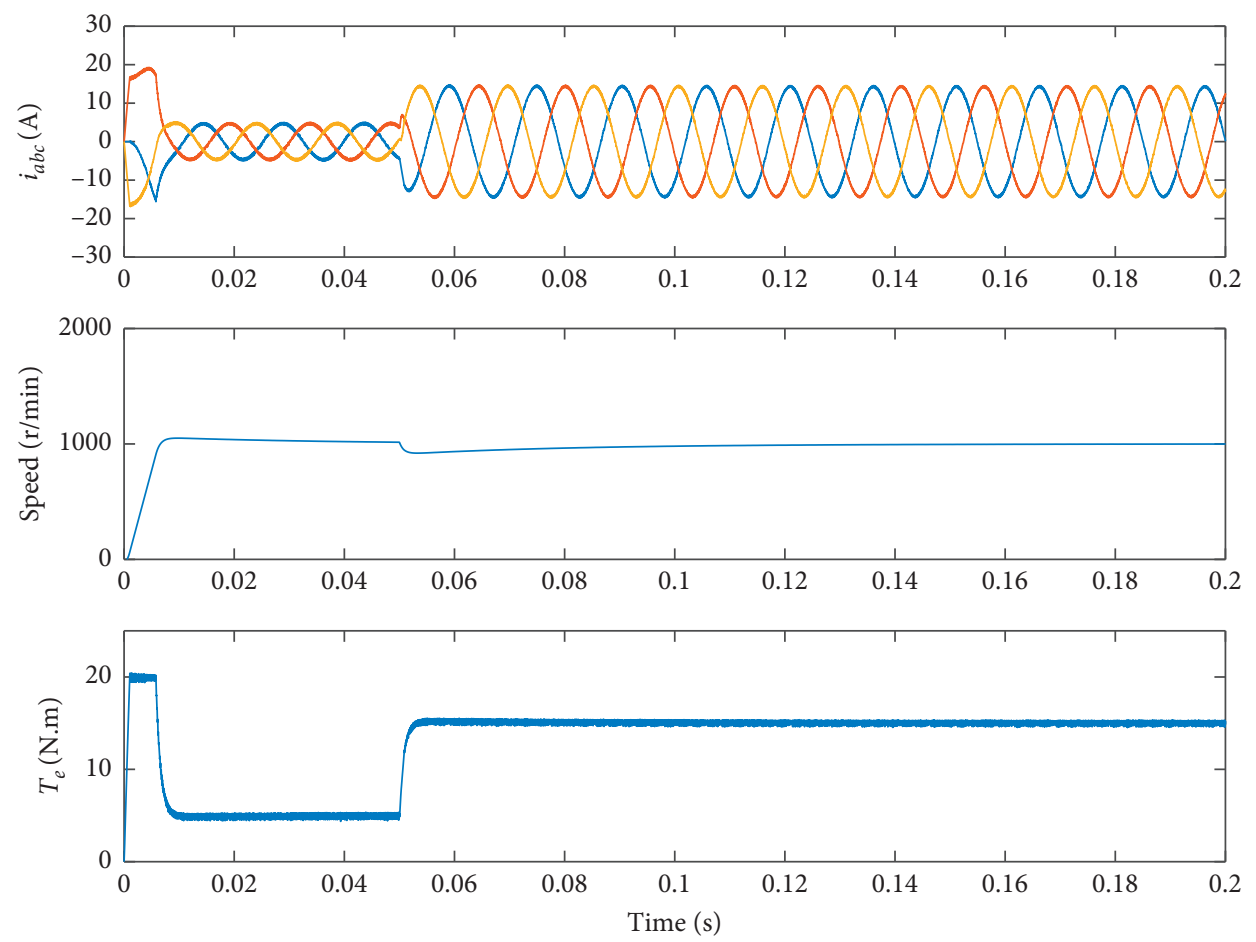

(a)

Figure 5: Continued. 

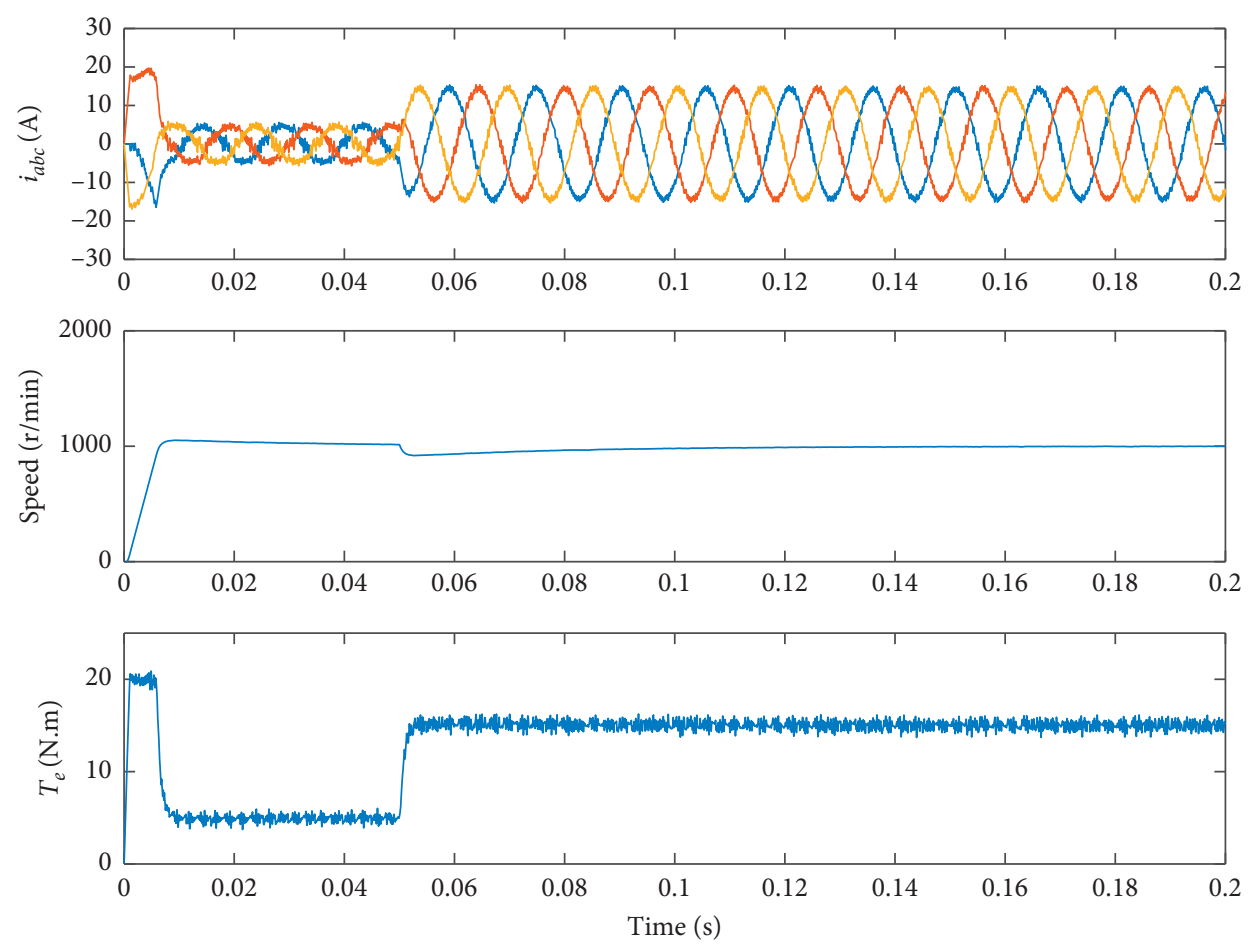

(b)
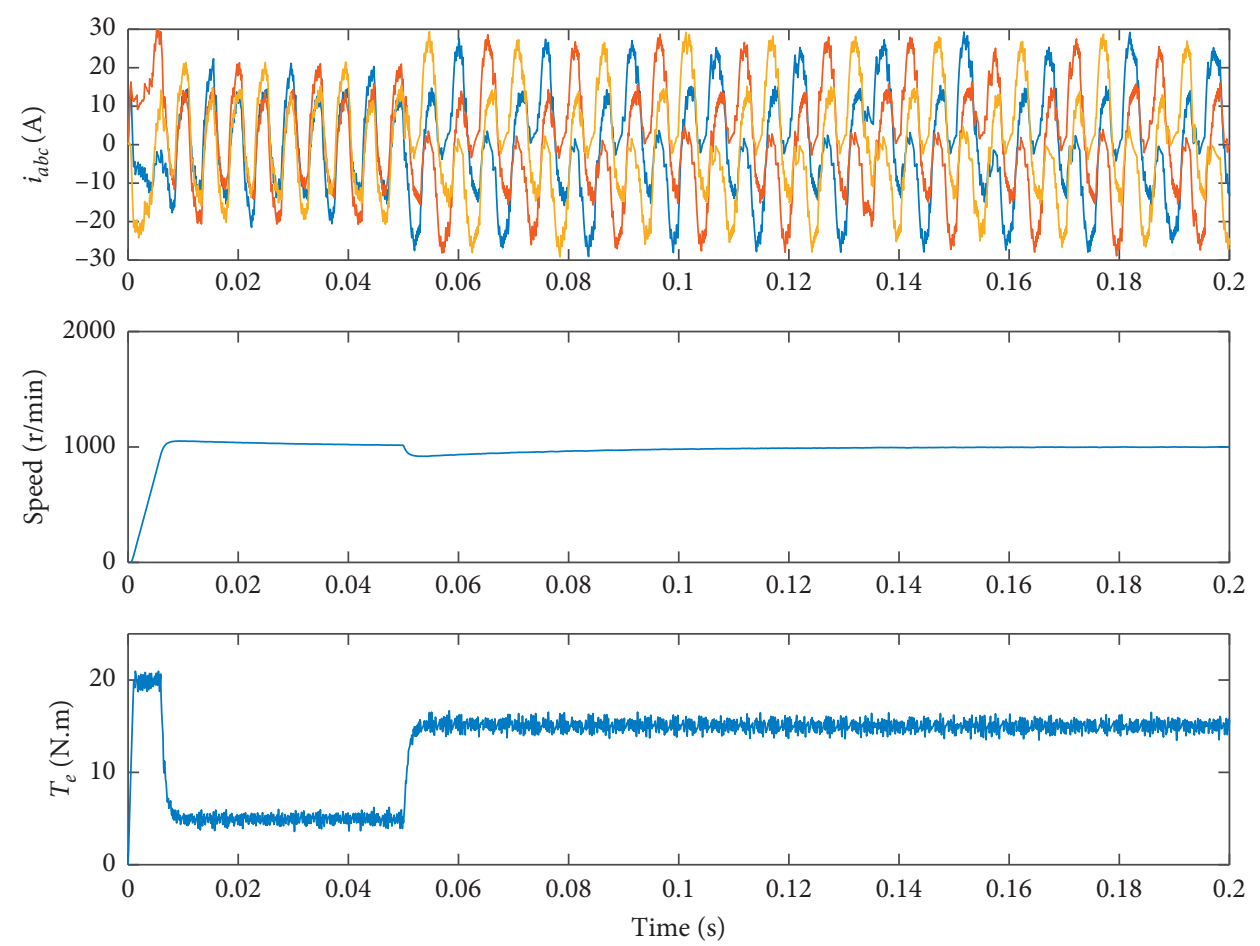

(c)

Figure 5: The result of phase current, speed, and torque. (a) Set-I. (b) Set-II. (c) Set-III.

voltage vectors and one zero vector from Figure 3 are selected as set-III for comparison as shown in Table 2.

In the simulation, the surface-mounted PMSM is taken as an example. The parameters of the PMSM are listed in Table 3. The speed is set to $1000 \mathrm{r} / \mathrm{min}$, and the common DC voltage is $150 \mathrm{~V}$. The initial torque is set to $5 \mathrm{~N} \mathrm{~m}$. Then, after 0.05 seconds, the torque is increased to $15 \mathrm{~N} \mathrm{~m}$. The sampling frequency is set to $100 \mathrm{kHz}$, and predictive control is performed every sampling period.

The simulation results are shown in Figures 5-7. 

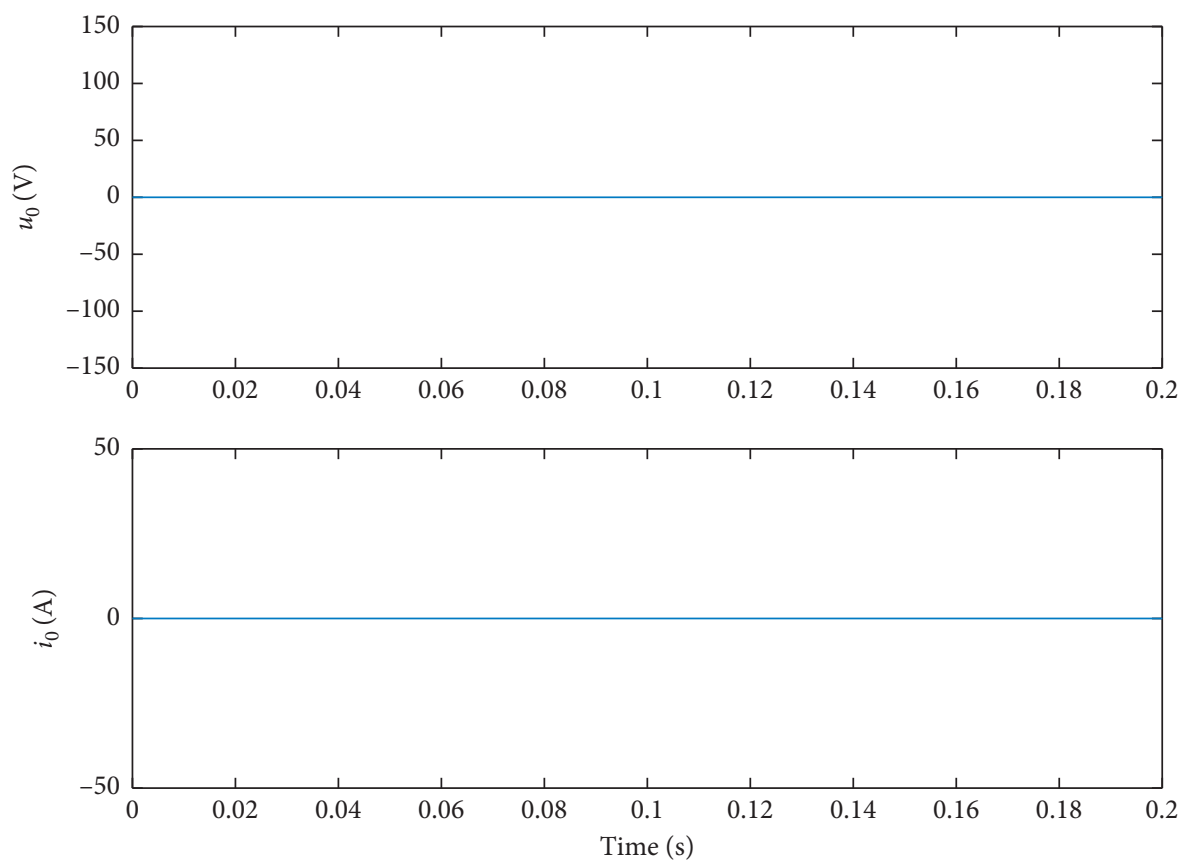

(a)
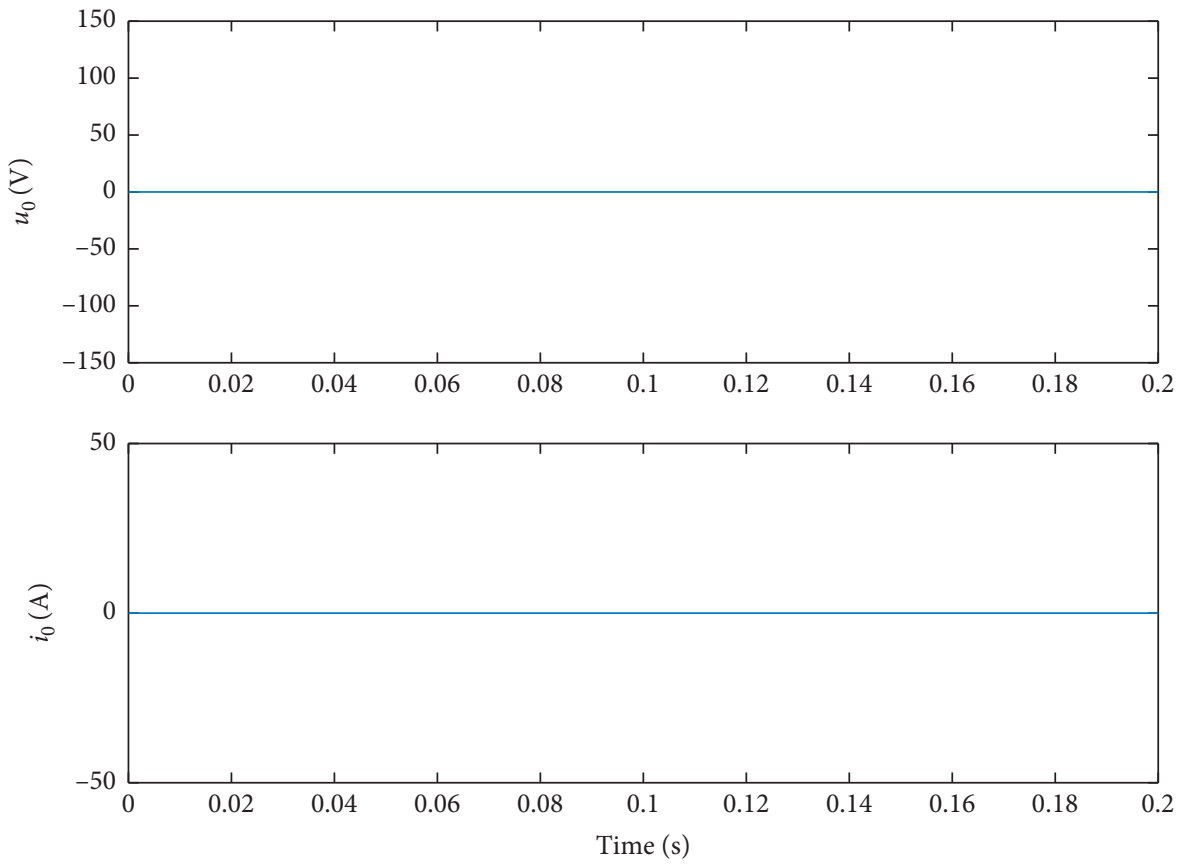

(b)

Figure 6: Continued. 

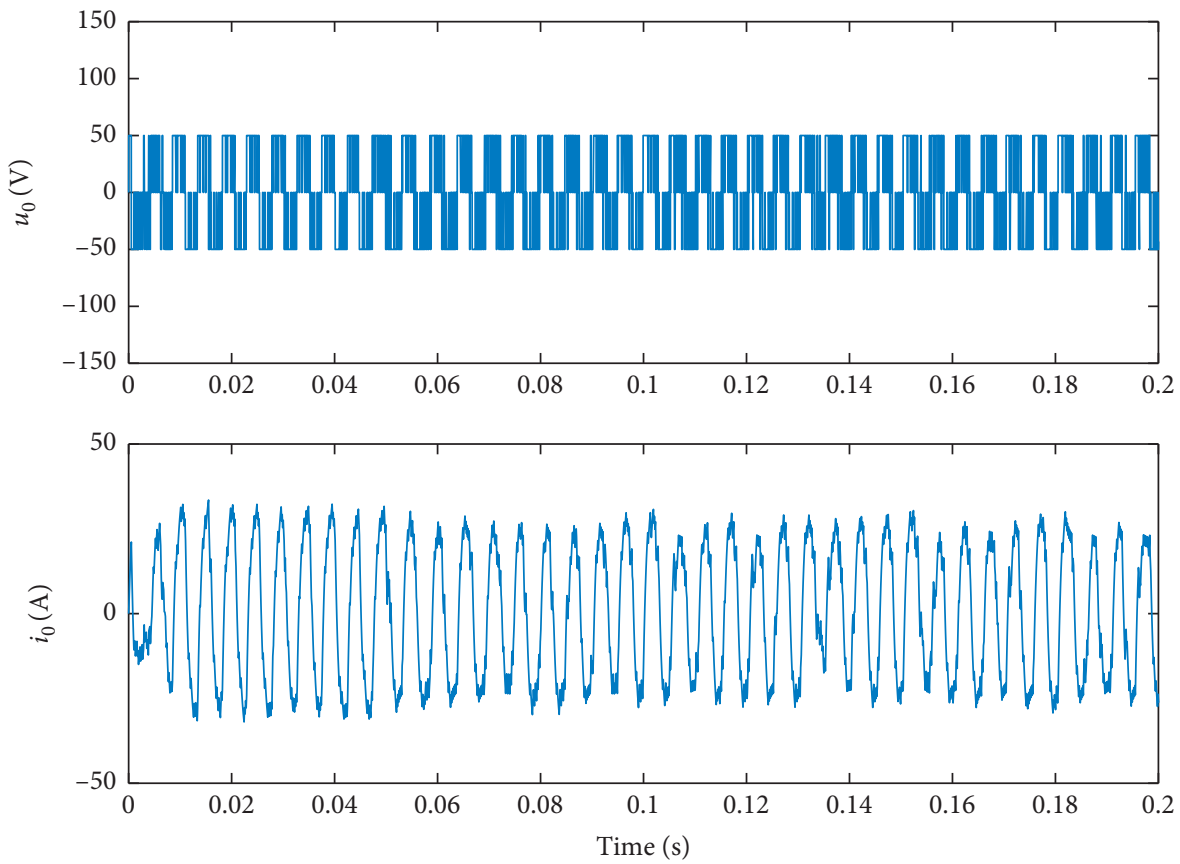

(c)

Figure 6: The result of $u_{0}$ and $i_{0}$. (a) Set-I. (b) Set-II. (c) Set-III.
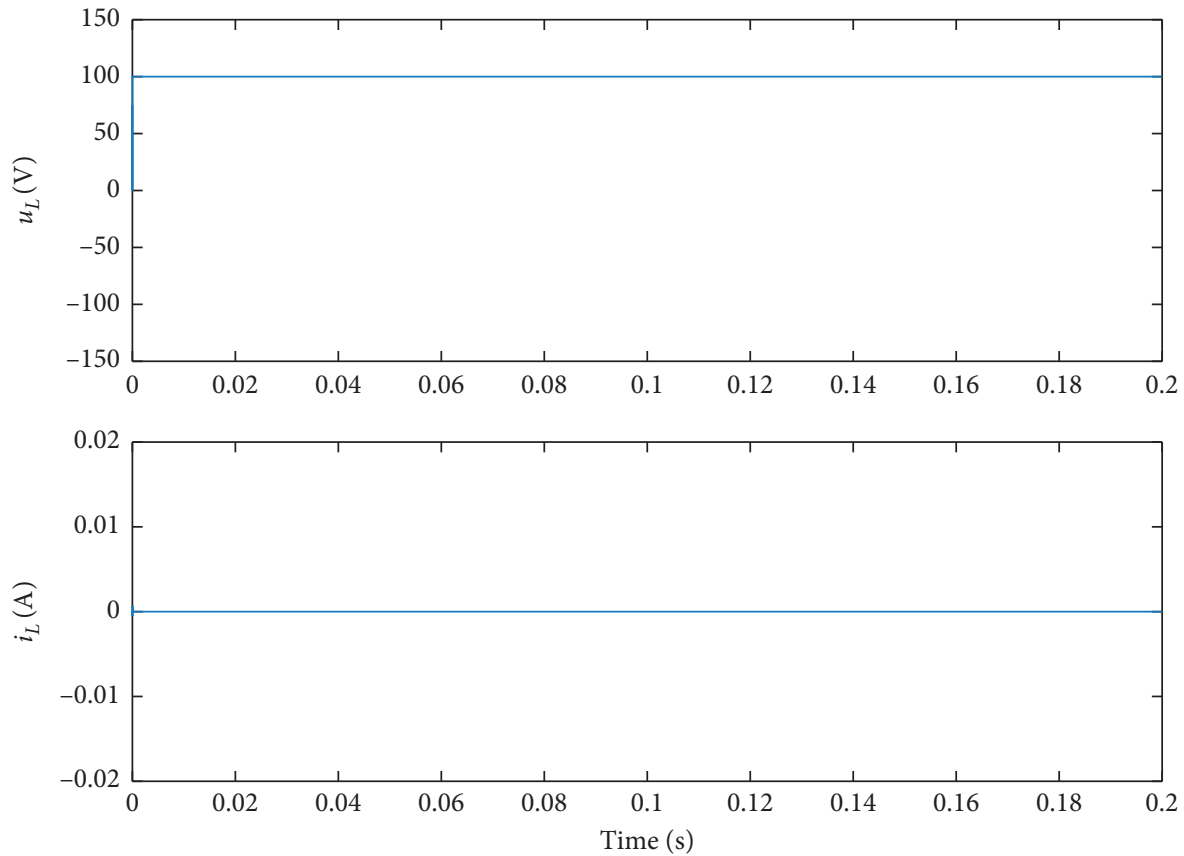

(a)

Figure 7: Continued. 

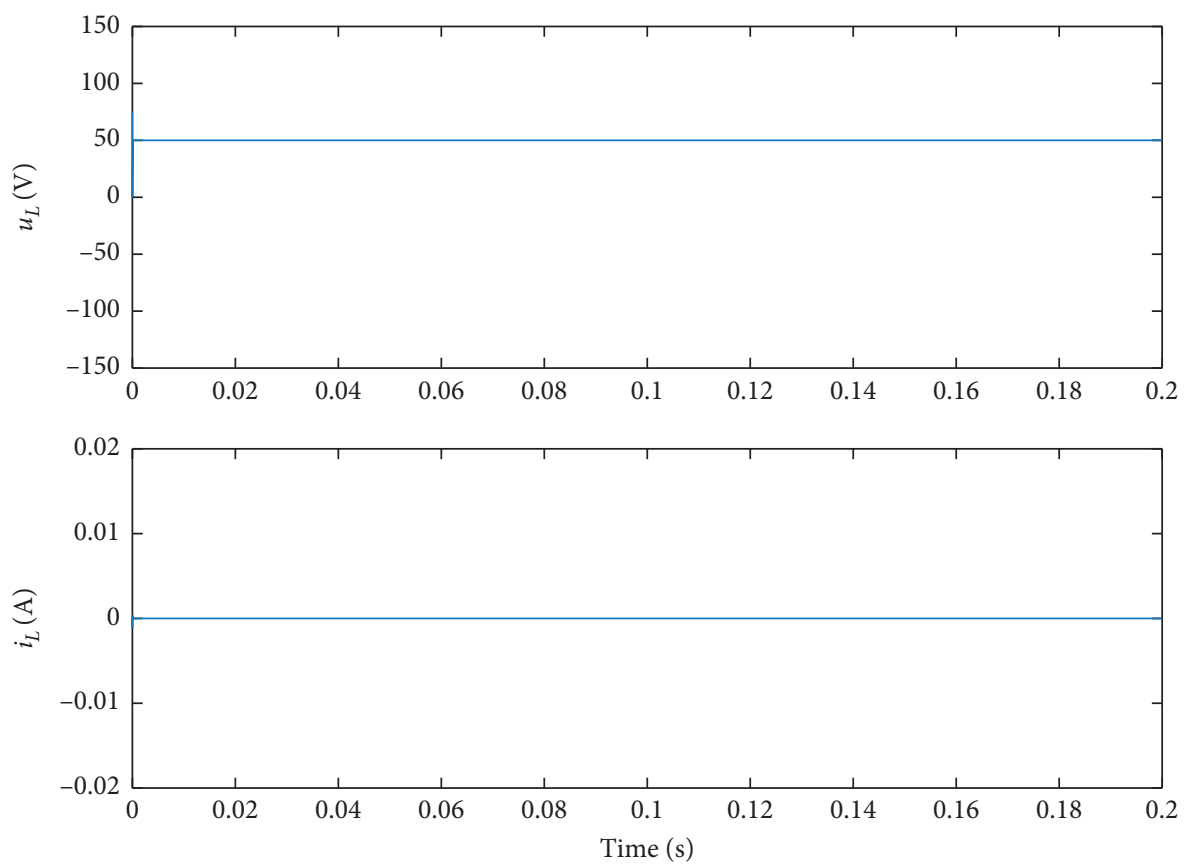

(b)
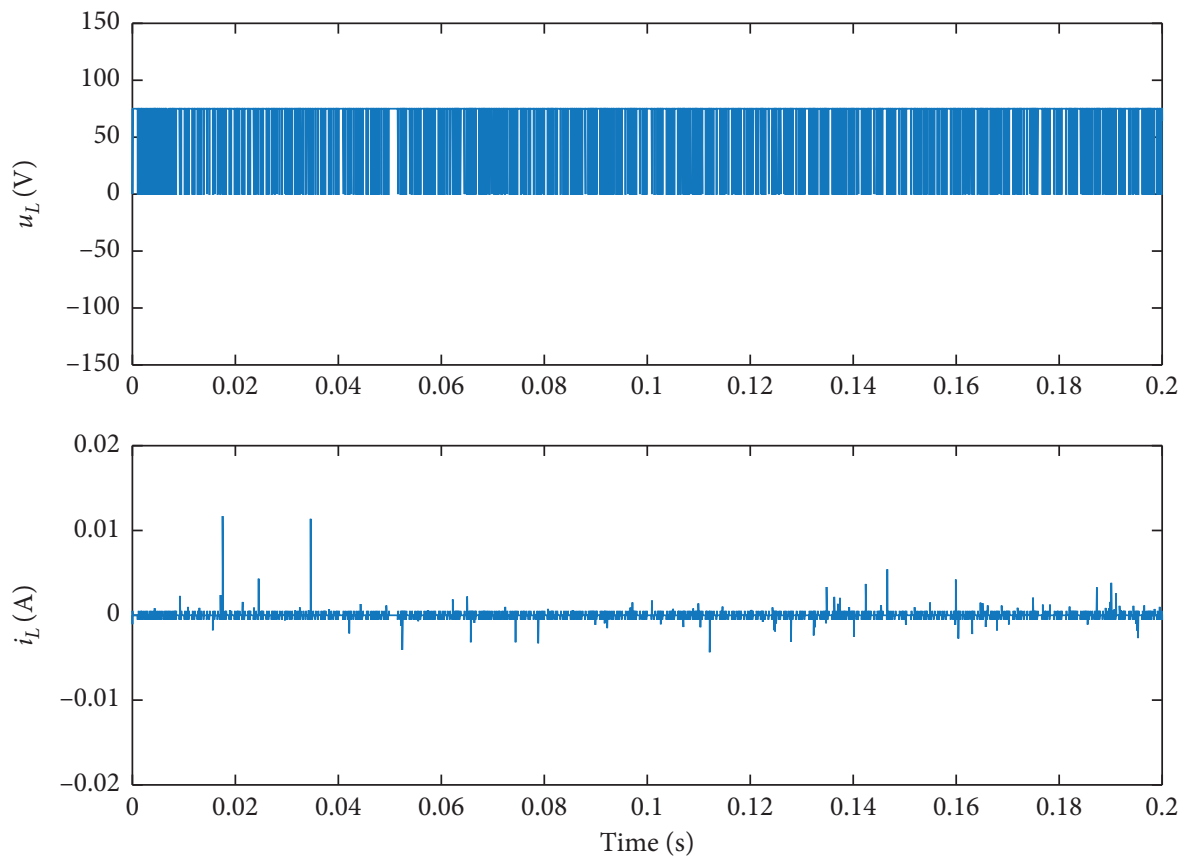

(c)

Figure 7: The result of $u_{L}$ and $i_{L}$. (a) Set-I. (b) Set-II. (c) Set-III.

It can be seen from Figure 5 that the dynamic response of speed and torque is fast for all sets. The phase current is smooth for set-I and set-II. However, the phase current of set-III is distorted, and it means there is some harmonic current included in this phase current.

$u_{0}$ of the three sets shown in Figure 6 is consistent with the analysis in Tables 1 and 2. Because $u_{0}$ of set-I and set-II is $0, i_{0}$ generated by set-I and set-II is also $0 . u_{0}$ of set-III varies between $-U_{\mathrm{dc}} / 3(50 \mathrm{~V})$ and $U_{\mathrm{dc}} / 3(50 \mathrm{~V})$, and $R_{s}$
$(1.2 \Omega)$ is small. Therefore, $i_{0}$ generated by set-III is large, and the phase current generated by set-III is distorted in Figure 5(c).

The results of $u_{L}$ shown in Figure 7 are also consistent with Tables 1 and 2 . For set-I and set-II, $u_{L}$ is $2 U_{\mathrm{dc}} / 3(100 \mathrm{~V})$ and $U_{\mathrm{dc}} / 3(50 \mathrm{~V})$, respectively, and the amplitude of $u_{L}$ is constant, which cannot generate current $i_{L}$. Therefore, $i_{L}$ is 0 in set-I and set-II. However, there exists $i_{L}$ in set-III because $u_{L}$ of set-III varies between $U_{\mathrm{dc}} / 2(75 \mathrm{~V})$ and 0 . 
In addition, in the simulation, the switching frequencies of the three schemes are counted. Sets I, II, and III are $77.56 \mathrm{kHz}, 77.77 \mathrm{kHz}$, and $53.44 \mathrm{kHz}$, respectively. Set-I and set-II are slightly smaller than the sampling period, and setIII is close to half of the sampling period.

From the figures, it can be seen that set-I and set-II can eliminate the influence of CMV on leakage current and zerosequence current in the OEW-PMSM. Compared with setIII, in set-I and set-II, the zero-sequence current $i_{0}$ can be reduced from the amplitude of $30 \mathrm{~A}$ to 0 , and the leakage current $i_{L}$ can be reduced from $10 \mathrm{~mA}$ to 0 . Moreover, set-II has smaller $u_{L}$ than that of set-I, but set-III has a smaller switching frequency.

\section{Conclusions}

For the OEW-PMSM system with common DC link, a MPTC method is proposed to eliminate the zero-sequence current and the leakage current caused by CMV. In this method, the voltage vector in the OEW-PMSM with the same CMV is selected to form the control vector sets. When the MPTC uses the vector sets to control the OEW-PMSM, the CMV of the zero-sequence current loop can be reduced to zero and the CMV of the leakage current loop can be kept constant. Therefore, the zero-sequence current and the leakage current caused by CMV can be eliminated without vector modulation. Meanwhile, the proposed cost function without the weight factor makes the distribution that there is no need to debug the weighting factor when deploying MPTC. Simulation results verify the feasibility and effectiveness of the method.

For the three-phase OEW motor system, the zerosequence loop consists of two inverters and stator winding, and the leakage current loop consists of stator winding and parasitic capacitive impedance. This paper takes the PMSM as the research object, but the proposed method can also be applied to the induction motor (IM) and PMSM with the same structure on the two loops.

\section{Data Availability}

All the data, together with relevant analysis scripts and files, are available from the corresponding author (e-mail: 52328173@qq.com) upon request.

\section{Conflicts of Interest}

The authors declare no conflicts of interest regarding the publication of this study.

\section{Acknowledgments}

The authors thank Dr. Dan Sun at Zhejiang University for her valuable comments on their manuscript. This work was supported in part by the Youth Fund of Zhejiang Provincial Natural Science Foundation (Grant no. LQ21F030002) and the Jiangsu Provincial Postdoctoral Science Foundation (Grant no. 2020Z230).

\section{References}

[1] B. A. Welchko, "A double-ended inverter system for the combined propulsion and energy management functions in hybrid vehicles with energy storage," in Proceedings of the 31st Annual Conference of IEEE Industrial Electronics Society, 2005 IECON 2005, Raleigh, NC, USA, November 2005.

[2] T. Kawabata, E. C. Ejiogu, Y. Kawabata, and K. Nishiyama, "New open-winding configurations for high-power inverters," in ISIE '97 Proceeding of the IEEE International Symposium on Industrial Electronics, pp. 457-462, Guimaraes, Portugal, July 1997.

[3] J. Korhonen, J. Honkanen, T. J. Kärkkäinen, J. Nerg, and P. Silventoinen, "Modulation and control methods to reduce zero sequence current in open-end winding motors," in Proceedings of the 2017 IEEE International Electric Machines and Drives Conference (IEMDC), pp. 1-6, Miami, FL, USA, May 2017.

[4] L. Yao, S. Yang, Z. Xie, and X. Zhang, "Eliminating scheme of zero-sequence currents in open-end winding induction motor drive with a single DC power supply," in Proceedings of the 2017 Chinese Automation Congress (CAC), pp. 3538-3543, Jinan, China, October 2017.

[5] J. M. Erdman, R. J. Kerkman, D. W. Schlegel, and G. L. Skibinski, "Effect of PWM inverters on AC motor bearing currents and shaft voltages," IEEE Transactions on Industry Applications, vol. 32, no. 2, pp. 250-259, 1996.

[6] F. Wang, "Motor shaft voltages and bearing currents and their reduction in multi-level medium voltage PWM voltage source inverter drive applications," in Proceedings of the Conference Record of the 1999 IEEE Industry Applications Conference Thirty-Forth IAS Annual Meeting (Cat. No.99CH36370), vol. 3, pp. 1602-1607, Phoenix, AZ, USA, August 1999.

[7] L. Rovere, A. Formentini, G. L. Calzo, P. Zanchetta, and T. Cox, "Zero-sequence voltage elimination for dual-fed common DC-link open-end winding PMSM high-speed starter-generator-Part II: deadtime hysteresis control of zerosequence current," IEEE Transactions on Industry Applications, vol. 55, no. 6, pp. 7813-7821, 2019.

[8] W. Hu, H. Nian, and D. Sun, "Zero-sequence current suppression strategy with reduced switching frequency for openend winding PMSM drives with common DC bus," IEEE Transactions on Industrial Electronics, vol. 66, no. 10, pp. 7613-7623, 2018.

[9] M. Cacciato, A. Consoli, G. Scarcella, and A. Testa, "Reduction of common-mode currents in PWM inverter motor drives," IEEE Transactions on Industry Applications, vol. 35, no. 2, pp. 469-476, 1999.

[10] M. C. Cavalcanti, K. C. de Oliveira, F. A. S. Neves, G. M. S. Azevedo, F. C. Camboim, and F. C. Camboim, "Modulation techniques to eliminate leakage currents in transformerless three-phase photovoltaic systems," IEEE Transactions on Industrial Electronics, vol. 57, no. 4, pp. 1360-1368, 2010.

[11] E. Un and A. M. Hava, "Performance analysis and comparison of reduced common mode voltage PWM and standard PWM techniques for three-phase voltage source inverters," in Proceedings of the Twenty-First Annual IEEE Applied Power Electronics Conference and Exposition, 2006. APEC, pp. 19-23, Dallas, TX, USA, March 2006.

[12] J. Kalaiselvi and S. Srinivas, "Bearing currents and shaft voltage reduction in dual-inverter-fed open-end winding induction motor with reduced CMV PWM methods," IEEE 
Transactions on Industrial Electronics, vol. 62, no. 1, pp. 144-152, 2015.

[13] L. Poh Chiang, D. G. Holmes, Y. Fukuta, and T. A. Lipo, "Reduced common-mode modulation strategies for cascaded multilevel inverters," IEEE Transactions on Industry Applications, vol. 39, no. 5, pp. 1386-1395, 2003.

[14] A. K. Gupta and A. M. Khambadkone, "A space vector modulation scheme to reduce common mode voltage for cascaded multilevel inverters," IEEE Transactions on Power Electronics, vol. 22, no. 5, pp. 1672-1681, 2007.

[15] H. Ghoreishy, A. Yazdian, S. Farhangi, and M. Mohamadian, "Reducing Common-mode voltage and power dissipation in cascaded multilevel inverters with flexible dc sources," in Proceedings of the 2010 1st Power Electronic \& Drive Systems \& Technologies Conference (PEDSTC), pp. 175-179, Tehran, Iran, February 2010.

[16] A. Muetze and C. R. Sullivan, "Simplified design of commonmode chokes for reduction of motor ground currents in inverter drives," IEEE Transactions on Industry Applications, vol. 47, no. 6, pp. 2570-2577, 2011.

[17] N. Zhu, J. Kang, D. Xu, B. Wu, and Y. Xiao, "An integrated AC choke design for common-mode current suppression in neutral-connected power converter systems," IEEE Transactions on Power Electronics, vol. 27, no. 3, pp. 1228-1236, 2012.

[18] J. Kalaiselvi and S. Srinivas, "Design and development of a single CM filter for bearing current and ground current reduction in a dual two level inverter fed open end winding induction motor drive," in Proceedings of the 2016 IEEE International Conference on Power Electronics, Drives and Energy Systems (PEDES), pp. 1-6, Trivandrum, India, December 2016.

[19] S. Gerber and R.-J. Wangi, "Reduction of inverter-induced shaft voltages using electrostatic shielding," in Proceedings of the 2019 Southern African Universities Power Engineering Conference/Robotics and Mechatronics/Pattern Recognition Association of South Africa (SAUPEC/RobMech/PRASA), pp. 310-315, Bloemfontein, South Africa, January 2019.

[20] M. Preindl and S. Bolognani, "Model predictive direct torque control with finite control set for PMSM drive systems, Part 1: maximum torque per ampere operation," IEEE Transactions on Industrial Informatics, vol. 9, no. 4, pp. 1912-1921, 2013.

[21] M. Norambuena, J. Rodriguez, Z. Zhang, F. Wang, C. Garcia, and R. Kennel, "A very simple strategy for high-quality performance of AC machines using model predictive control," IEEE Transactions on Power Electronics, vol. 34, no. 1, pp. 794-800, 2019.

[22] Z. Zhang, A. Chen, X. Xing, and C. Zhang, "A novel model predictive control algorithm to suppress the zero-sequence circulating currents for parallel three-phase voltage source inverters," in Proceedings of the 2016 IEEE Applied Power Electronics Conference and Exposition (APEC), pp. 3465-3470, Long Beach, CA, USA, March 2016.

[23] X. Wang, J. Zou, J. Zhao et al., "A novel model predictive control strategy to eliminate zero-sequence circulating current in paralleled three-level inverters," IEEE Journal of Emerging and Selected Topics in Power Electronics, vol. 7, no. 1, pp. 309-320, 2019. 